\title{
Prostitution et Vox literati à Shangaï avant la Première Guerre mondiale
}

\section{Christian HENRIOT}

\section{(2) OpenEdition \\ 1 Journals}

\section{Édition électronique}

URL : https://journals.openedition.org/clio/581

DOI : 10.4000/clio.581

ISSN : 1777-5299

Éditeur

Belin

\section{Édition imprimée}

Date de publication : 1 avril 2003

Pagination : 45-64

ISBN : 2-85816-663-3

ISSN : 1252-7017

Référence électronique

Christian HENRIOT, «Prostitution et Vox literati à Shangaï avant la Première Guerre mondiale », Clio. Histoire, femmes et sociétés [En ligne], 17 | 2003, mis en ligne le 27 novembre 2006, consulté le 24 avril 2022. URL : http://journals.openedition.org/clio/581 ; DOI : https://doi.org/10.4000/clio.581

Ce document a été généré automatiquement le 24 avril 2022.

Tous droits réservés 


\title{
Prostitution et Vox literati à Shangaï avant la Première Guerre mondiale
}

\author{
Christian HENRIOT
}

1 Le phénomène de la prostitution a engendré en Occident depuis le Moyen Âge un souci assez constant, bien que plus ou moins formalisé, de surveillance, d'encadrement et de répression. Intimement lié à la sexualité, il a été l'objet depuis cette époque d'une multiplicité de discours s'entrecroisant et se renforçant pour créer dans les esprits et les mentalités une attitude croissante de rejet et de condamnation. C'est au XIXe siècle que l'encerclement de la prostitution dans un faisceau de discours et de mesures médico-policières connaît une sorte d'apogée. Il est lié à la convergence de plusieurs facteurs anciens et nouveaux - condamnation religieuse et morale de la prostitution, souci de surveillance des pouvoirs publics, anxiété à l'égard des maladies vénériennes pour ne citer que les principaux - dans un contexte social et idéologique particulier, marqué par une crainte des élites bourgeoises d'être socialement et physiquement gangrenées par une prostitution non contrôlée issue des classes prolétaires. Cet état d'esprit a permis à certains acteurs, notamment le corps médical, d'avoir une influence déterminante sur l'attitude des pouvoirs publics à l'égard des prostituées et, sous couvert d'expertise scientifique, de nourrir un débat foncièrement biaisé.

2 En Europe, les formes qu'ont prises les mesures d'encadrement ou de répression de la prostitution ont été moulées par les traditions différentes au plan de la religion, de la structure sociale et de l'organisation du pouvoir des divers pays du continent. Toutefois, on retrouve dans chacun d'eux les mêmes fils conducteurs qui ont servi de trame à la mise en place de l'appareil répressif qui tente d'endiguer ou d'envelopper le phénomène prostitutionnel. L'une des hypothèses de travail de cette étude était à l'origine d'examiner si la Chine avait connu un phénomène parallèle et si la société chinoise avait produit, à l'époque prise en compte ici, des formes spécifiques de discours sur la prostitution. Force est de constater que si l'on relève des éléments d'expression sur ce sujet, l'ensemble est fragmentaire, informe, peut-être par manque de "locuteurs", mais surtout par suite d'une absence de condamnation véritable de la prostitution et de prise de conscience de ses effets. Il faut y ajouter, jusqu'à la Première 
Guerre mondiale, le poids de la domination d'un discours "positif", bien que fallacieux, des élites lettrées sur les courtisanes. Enfin, il devient rapidement très difficile de faire la part des réactions "autochtones" et des influences occidentales qui se manifestent dès 1869 à Shanghai.

L'étude des formes de discours sur la prostitution constitue un domaine largement inexploré. Trois sources principales peuvent être identifiées, qui ont contribué à former l'attitude de la société et du pouvoir à l'égard des prostituées. La première est le droit, c'est-à-dire les codes juridiques sous l'Empire et la République qui permettent de prendre la mesure du degré d'intérêt que les gouvernants chinois ont accordé à la prostitution. La seconde rassemble les opinions exprimées par des membres de l'élite lettrée dans leurs écrits personnels et dans des lettres adressées aux journaux. La troisième représente une vaste entité, la littérature, que je n'ai fait qu'effleurer. J'ai examiné le discours juridique et littéraire dans la perspective de l'historien dans mon travail initial. Dans cet article, je n'aborderai que le deuxième champ, celui des écrits de l'élite lettrée. La période concernée est principalement le XIXe siècle, même si de larges incursions sont menées dans le siècle suivant. C'est une période qui voit la persistance d'une vision favorable, quoique tronquée, de la prostitution et le début d'un infléchissement de la sensibilité des élites dans le sens d'un discours de plus en plus critique.

4 L'opinion publique, au XIXe siècle, se résume aux élites lettrées qui ont la maîtrise de l'écrit et qui sont les seules à disposer des moyens de diffuser leurs idées au sein de la société. Ces élites s'attachent à leur statut, un prestige qui leur donne des pouvoirs et une influence très larges sur la population, en particulier dans le domaine "idéologique". Elles sont non seulement les détentrices du savoir, mais aussi les garantes d'un ordre politique et moral qu'elles confortent et nourrissent par leurs actions et leurs écrits. Néanmoins, même si elles jouaient un rôle crucial au sein de la société, elles n'étaient pas responsables formellement du contrôle des mœurs ou de la police sanitaire à la manière de leurs contemporains en Europe. Avant l'arrivée des Occidentaux, l'opinion des lettrés sur la prostitution s'exprimait tout d'abord dans les mémoires et écrits divers qu'ils ont laissés sur ce sujet précis ou sur leurs expériences de voyage. Les raisons de leur choix sont parfois exprimées explicitement, mais le plus souvent ils se contentent de rapporter des anecdotes mêlées d'informations concrètes, bien qu'invérifiables, et de considérations morales. En fait, le genre dans lequel les lettrés s'expriment "donne le ton ». Il fournit le langage et les images qui traversent les textes. La lecture de ces ouvrages était circonscrite pour l'essentiel à l'élite lettrée, mais le discours que l'on y trouve a plus largement transpiré "vers le bas" et contribué à imprégner le corps social. Finalement, les lettrés s'intéressaient à la couche supérieure des prostituées, c'est-à-dire les courtisanes.

5 L'émergence de la presse d'information a permis à des opinions plus variées de s'exprimer, notamment sur la prostitution en tant que problème social, une dimension qui n'était guère présente dans les écrits antérieurs. Les journaux ne représentent pas seulement un nouveau support. Ils créent un domaine entièrement nouveau de représentations. C'est une innovation qui se développe dans un très petit nombre de lieux en Chine. Même si cette avancée sert initialement des objectifs politiques, elle entraîne l'apparition d'une presse d'information dont le Shen Bao est le meilleur exemple ${ }^{1}$. Elle s'accompagne aussi d'une véritable explosion de l'édition qui fournit à son tour un nouveau domaine en termes d'emplois et d'expression pour les lettrés. Ce 
nouveau contexte a créé les conditions de l'émergence d'un nouveau type de lettrés qui, parce qu'ils sont détachés de la voie traditionnelle des examens impériaux et de la recherche de postes officiels au sein de l'administration impériale, se tournent vers l'écriture comme métier permanent et forment le terreau initial de formation des intellectuels modernes du XXe siècle ${ }^{2}$. La dimension de critique sociale était assez peu présente dans les écrits littéraires antérieurs. Ce discours "lettré" a déterminé la conception "chinoise" de la prostitution qui s'est imposée à la société et aux historiens qui ont abordé cette question. Or le message que véhiculent ces écrits est ambivalent. Il $\mathrm{y}$ a de la part des lettrés une sorte de double langage qui tend à brouiller la manière dont la prostitution était réellement perçue par la société. Quel était ce discours dominant?

Le discours ambivalent des lettrés

6 J'examinerai tout d'abord la série d'ouvrages écrits par Wang Tao, en particulier Haizou yeyou, et m'efforcerai de mettre en lumière l'opinion des lettrés sur la prostitution ${ }^{3}$. Dans son introduction, Wang pose clairement qu'il a écrit ce recueil pour laisser à la postérité une trace de la vie des courtisanes dont il reste généralement peu de choses. Il se réfère à d'autres ouvrages semblables publiés dans le passé comme Banqiao zaji (Notes diverses du pont de bois) ou Wumen huafang lu (Récit des lupanars de Suzhou) ${ }^{4}$. C'est en effet une tradition bien enracinée chez les lettrés de brosser des portraits de courtisanes célèbres qu'ils ont fréquentées, même si la valeur de ces écrits en tant que source historique est limitée ${ }^{5}$. Ils sont cependant en eux-mêmes très révélateurs de l'attitude des lettrés à l'égard de cette catégorie particulière de femmes. Les termes employés pour les qualifier et pour les décrire sont toujours très laudatifs. Wang Tao n'utilise jamais le mot de "prostituée". Les courtisanes sont évoquées par des métaphores littéraires ou des périphrases. Même les rares allusions à leur condition parfois difficile ne vont pas au-delà de l'expression "mer d'amertume" (kuhai). Il précise aussi que dans le "royaume des fleurs" (huaguo), il n'y a pas de femmes de basse catégorie.

7 On n'y trouve pas non plus de réflexion sur le destin des courtisanes. Wang ne fait qu'effleurer la question par une simple énumération des circonstances qui ont pu amener ces femmes à la prostitution: élevées dans ce milieu, contraintes par la pauvreté, par un mariage raté, orphelines.... Mais cela importe peu au fond car elles sont là, pour l'agrément des élites, et l'auteur ne veut connaître d'elles que cette étape de leur vie qu'il encense. Wang ne se pose pas la question de savoir comment elles peuvent se sentir. Il ne dit rien ou presque de leurs misères quotidiennes ou accidentelles, à l'exception des maladies d'origine sentimentale ou des suicides. La dimension matérielle est presque totalement occultée. Le "royaume des fleurs" apparaît comme une sorte de paradis où les hommes de culture peuvent rencontrer des femmes de talent même si celles-ci ont rarement un niveau d'éducation très élevé. Ce qui importe toutefois, c'est cette image d'un lieu intemporel - il serait intéressant de faire une étude comparée des portraits de courtisanes écrits sous différentes dynasties sur lequel Wang comme d'autres lettrés portent un regard de Candide. C'est dire que de la prostitution émerge une image complètement biaisée qui ne rend compte ni de la réalité, ni de la perception réelle du phénomène prostitutionnel.

8 Wang Tao n'est pas exceptionnel ${ }^{6}$. Outre les ouvrages antérieurs mentionnés précédemment, on retrouve chez ses contemporains de nombreux témoignages semblables. Dans ses souvenirs d'un séjour à Shanghai, Chi Zhicheng, un lettré résidant 
à Taiwan, se rémémore une ville animée et bruyante que sillonne le soir un flot continu de courtisanes décrites en des termes laudatifs ${ }^{7}$. De nombreux lettrés ont consacré tout ou partie de leurs mémoires ou récits au milieu des prostituées. Bien que je ne les aie pas lus, faute de les avoir retrouvés, ils s'inscrivent dans cette même veine de textes qui cherchent à transmettre une vision positive de l'élite des prostituées ${ }^{8}$. Dans un autre genre, de nombreux ouvrages ont été publiés sur ce groupe de prostituées sous la forme d'illustrations accompagnées d'un court commentaire destiné à éclairer la personnalité de chaque fille présentée. Une bonne partie de ces livres est parue entre le milieu des années 1880 et le début des années $1890^{\circ}$. Mes recherches à Shanghai ont permis de localiser une infime proportion de cette littérature qui, une fois encore, n'apporte guère d'éléments concrets et fiables, mais qui révèle l'estime dans laquelle étaient tenues les courtisanes ${ }^{10}$. Le Haishang qinglou tuji (Notes et gravures des pavillons verts de Shanghai) n'est qu'un exemple de cet engouement des lettrés pour un milieu qui les fascine et dont ils se font les hérauts ${ }^{11}$.

Peut-on pour autant considérer que la prostitution en tant que phénomène social n'est pas perçue de façon négative par les lettrés ? Jugent-ils cette activité comme normale, acceptable ou inévitable? Opèrent-ils une distinction entre différents niveaux de prostituées ? La réponse à ces questions pourrait tenir en quelques mots : les lettrés ne parlent tout simplement pas des prostituées autres que les courtisanes. Les catégories inférieures sont parfois évoquées brièvement, mais elles sont le plus souvent oubliées, occultées, repoussées dans la pénombre qui enveloppe l'histoire des couches populaires. Il y a des exceptions, cependant, comme les ouvrages de Wang Tao qui ont permis d'éclairer partiellement la prostitution populaire au milieu du XIXe siècle. Or que dit-il des filles qui, pour parodier l'expression appliquée aux courtisanes, « vendent leur corps et non leur art »? Il décrit leur monde comme une mer de souffrance habitée par des démons. Il n'y a plus de métaphores littéraires. Les filles sont décrites sous le jour le plus noir : âgées, excessivement maquillées, échevelées et mal vêtues, elles sont destinées à devenir des "squelettes poudreux $»^{12}$. En 1891, le regard que porte Chi Zhicheng n'est pas différent. Il met dans le même sac yeji, huayanjian, prostituées âgées dont il dit qu'elles ont mauvaise réputation. Lui-même est allé les voir de loin, de sa chaise à porteur, et rapporte qu'il a cru entrer dans le royaume des démons

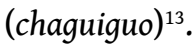

On constate donc, à la lumière de ces deux exemples, que les lettrés abhorrent au fond d'eux-mêmes cette prostitution populaire pour laquelle ils n'ont que du mépris. Cette attitude est à mettre en relation avec la condescendance avec laquelle ils traitent en général le menu peuple, perçu comme inculte ou ignorant, désordonné et grossier, et qu'il faut constamment encadrer et rappeler à l'ordre. Toutefois, dans le cas des prostituées, cette condescendance va jusqu'au dégoût, bien qu'il soit rarement exprimé et qu'il ne soit pas fondé sur des considérations morales liées à la sexualité. Comme on le verra plus loin dans l'analyse des courriers qu'ils adressent à la presse, ce n'est pas tant la promiscuité sexuelle que condamnent les lettrés que les prostituées comme facteur de désordre social, en particulier leur conquête de la rue et leurs pratiques indécentes de racolage. Dans leurs écrits, qu'il s'agisse de mémoires ou d'ouvrages littéraires, les élites lettrées ont préféré tout simplement ignorer ces catégories et réserver leur prose à celles qui ont la charge de les distraire, créant ainsi, avant l'introduction de la presse moderne, une formidable distorsion du discours sur laquelle je reviendrai in fine. 
La presse et la naissance d'une nouvelle conscience

11 La presse a introduit un nouveau media à travers lequel toutes sortes d'individus ont pu exprimer leurs idées, revendications et protestations. À Shanghai, le Shen Bao est devenu très tôt la principale plate-forme de discussion des questions politiques et sociales. C'est dans les toutes premières années de publication du Shen Bao que l'on trouve un certain nombre d'articles ou de lettres de lecteurs s'exprimant sur la question de la prostitution. Je n'ai pas vraiment d'explication à ce phénomène sinon qu'il est sans doute lié à la décision de l'intendant de circuit de Shanghai d'interdire les maisons de prostitution dans les concessions. Bien qu'il se soit avéré impuissant à faire respecter son arrêté, cela ne l'a pas empêché, au cours de ces années d'exercer différentes formes de pression sur les établissements concernés pour leur faire quitter leur havre. Le développement rapide de la prostitution à Shanghai et la mutation de ses formes ont sans doute aussi contribué à l'expression d'opinions variées sur ce phénomène, bien que j'aie trouvé peu d'articles dans les dernières décennies du siècle. Hormis les faits divers et les rapports des cours mixtes, la prostitution en tant que problème social ne surgit finalement dans la presse qu'à l'occasion d'interventions du pouvoir. Elle n'est à aucun moment le centre d'un véritable débat ${ }^{14}$. Les lecteurs qui adressent des courriers au Shen Bao appartiennent de manière évidente au milieu des lettrés comme en témoignent les formes parfois très élaborées dans lesquelles sont rédigées leurs missives.

12 Le premier texte relevé est une proposition de solution définitive au problème de la prostitution : éliminer les prostituées en leur permettant de s'affranchir (tushen) sous le contrôle des autorités. Les filles pourraient être rachetées par leur famille, par un client ou par elles-mêmes à un prix dégressif selon la catégorie, de 10 à 30 taëls, une somme très inférieure aux montants généralement versés pour le rachat d'une prostituée ou d'une courtisane. Ces lettres font explicitement référence aux prostituées ordinaires. Chaque ancienne prostituée se verrait remettre un certificat d'identité portant sa photo, un nouvel élément de modernité. De cette manière, les filles pourraient échapper à leur condition et effacer leur passé ${ }^{15}$. L'idée est généreuse, mais elle révèle une méconnaissance profonde du milieu de la prostitution et de grandes illusions sur la volonté et la capacité des autorités à mettre en œuvre un tel plan. Un autre lecteur a repris la même proposition, en exprimant son accord sur l'objectif et la méthode, mais il craint que l'interdiction de la prostitution ne serve de rien si l'avenir ou la subsistance des filles ne sont pas garantis. Il note qu'un bon nombre de femmes venues se réfugier à Shanghai au moment des troubles liés aux Taiping sont devenues prostituées faute de moyens pour vivre ${ }^{16}$.

13 On pourrait rattacher au point de vue exprimé plus haut les appels au mariage comme moyen de sortie du métier. Les magistrats chinois mettaient littéralement en vente (adjudication) des prostituées en échange du paiement d'une somme d'argent, sans demander l'avis des intéressées ${ }^{17}$. Dans la même veine, l'abolition de l'esclavage en Chine en 1906 exigeait des propriétaires d'esclaves de marier toutes leurs domestiques avant de les affranchir ${ }^{18}$. Une lettre de lecteur, qui ne prend pas position sur l'opportunité d'éliminer la prostitution ou sur sa place dans la société, appelle les prostituées à se marier, même avec un homme de condition modeste. C'est une assurance pour l'avenir, une fois passée leur beauté éphémère. Le texte, rédigé en imitation d'un texte connu de Tao Yuanming de l'époque des Six dynasties (220-589), décrit de façon détaillée tous les avantages de la vie d'épouse et de mère de famille ${ }^{19}$. 
Cet avis n'est pas partagé par un autre auteur qui montre comment les courtisanes se livrent à toutes sortes d'artifices pour séduire les clients dans le but de se faire épouser. Une fois leur objectif réalisé, elles s'empressent de prendre la fuite, en emportant argent, vêtements et bijoux. Le mari déconfit n'a plus qu'à se tourner vers la police et à affronter les regards de réprobation de sa famille, sans compter les maladies qu'il aura $\mathrm{pu}$ contracter auprès de la prostituée et qu'il aura transmises à son épouse ${ }^{20}$. Cet avis direct mettait dans un même sac prostituées et courtisanes comme catégorie à risque pour la santé physique.

Un autre lecteur suggère de s'attaquer au problème de la prostitution en amont, c'està-dire en éliminant les trafiquants d'êtres humains qui approvisionnent les maisons. Selon cet auteur, l'interdiction de la prostitution est un sujet évoqué depuis les années 1820 , sans résultat ${ }^{21}$. Le problème est ancien, mais il n'est pas insoluble. L'auteur préconise la peine capitale pour les vendeurs et les acheteurs ${ }^{22}$. Le détournement et la vente de jeunes filles est aussi au centre d'une lettre qui, bien que portant sur un sujet d'actualité, est rédigée sur le mode d'un écrit célèbre de Luo Binwang sous la dynastie des Tang (618-907). L'auteur dénonce les vieilles concubines abandonnées qui recueillent ou attirent des jeunes filles de bonne famille ou de famille pauvre pour les pervertir. Il évoque les mesures prises par l'intendant de circuit, mais il pense qu'elles sont insuffisantes tant que les hommes qui fréquentent les prostituées ne renoncent pas à la débauche. L'auteur en appelle à leur discernement, sur les risques encourus, et à leur vertu pour qu'ils se protègent eux-mêmes et préservent l'honneur des femmes ${ }^{23}$.

Les lettres précédentes portent plus sur les moyens d'éliminer la prostitution, sans distinction de catégories, mais elles ne nous éclairent guère sur les raisons qui motivent ces propositions. Dans une contribution rédigée sur un mode littéraire particulier (piantiwen), un lettré appelle à la suppression des maisons de prostitution ${ }^{24}$. Les deux points qu'il met en avant dans ce texte sont le constat d'une extension du phénomène prostitutionnel dans les concessions, en particulier dans les lieux publics, et les contacts de l'intendant avec les consuls étrangers pour remédier à cette situation. Son appel à la fermeture se fonde sur l'idée que la présence accrue des prostituées crée un désordre "moral" et nuit à la vie du pays. Le point de vue de l'auteur sur les moyens à mettre en œuvre est imprécis, bien qu'il conclue sur une incitation aux autorités à se montrer fermes. Il pense que les gens raisonnables savent le bénéfice à attendre d'une interdiction de la prostitution et s'y conformeront, mais il faudra éduquer les ignorants et éventuellement leur appliquer toute la rigueur de la loi ${ }^{25}$. Le désordre "moral" qu'invoque l'auteur n'est autre que ce qu'il perçoit comme un désordre "social", celui que causent les liuji, ces prostituées "mobiles" qui pratiquent le racolage sur la voie publique.

16 En effet, lorsque le terme de prostitution qu'emploient les lettrés dans leurs courriers est défini plus précisément, il s'agit toujours des prostituées de basse catégorie. Les huayanjian sont ainsi la cible d'une lettre qui juge que ces maisons offrent le pire mélange possible, prostitution et opium. C'est toutefois plutôt l'incitation à fumer qui est faite aux clients et qui les transforme en drogués que condamne l'article. Les filles sont néanmoins en cause car elles servent d'appât. L'auteur met aussi l'accent sur les maladies vénériennes dont sont atteintes les prostituées de ces établissements, qualifiées de "démons des maladies de peau". Il met en garde contre les risques certains d'infection et ses conséquences pour l'individu et sa descendance ${ }^{26}$. C'est aussi l'interdiction que préconise un autre article qui se préoccupe des conséquences de la 
prostitution. Il constate que les filles ne s'intéressent qu'à l'argent - tout le reste est factice - et emploient tous leurs moyens de séduction pour faire perdre la tête aux clients. Il en résulte souvent la ruine financière et physique de l'individu qui perd santé, famille et amis. L'auteur ne propose cependant aucun moyen précis pour parvenir à l'élimination de la prostitution. Il préconise simplement aux hommes de ne plus fréquenter ces établissements qui disparaîtront ainsi d'eux-mêmes ${ }^{27}$. C'est donc aussi la dimension de désordre social, centrée cette fois sur la famille, qui est mise en avant. Les maladies vénériennes sont mentionnées mais elles ne constituent pas un élément central de l'argumentation ${ }^{28}$. Le plus intéressant est cet appel à la vertu, invoquée dans une autre lettre, pour provoquer l'extinction de la prostitution.

Dans des textes plus tardifs, la demande d'interdiction des maisons de prostitution est liée à l'accroissement de la criminalité. Selon un lecteur qui écrit en 1889, la prostitution favoriserait ou engendrerait des conduites criminelles, notamment toute une petite délinquance qui affecte la population, victime de rapines dont les voleurs vont dépenser le produit dans les maisons de prostitution ${ }^{29}$. Dix ans plus tard, un article pointe spécifiquement les huayanjian, assimilés à des lieux réunissant tous les vices (prostitution, opium et jeu) fréquentés par des gens honnêtes (liang) mais aussi par des voyous auxquels ces établissements servent de refuge. Il faut donc les interdire si l'on veut voir baisser la criminalités ${ }^{30}$. Ces deux contributions soulignent aussi le souci des élites chinoises pour tout ce qui apparaît comme facteur de désordre au sein de la société. Il serait imprudent d'interpréter cela comme la montée d'un discours sur les "classes dangereuses", même si d'autres sources soulignent l'accroissement de la criminalité. On a vu que les maisons de prostitution, en particulier celles des courtisanes, en sont les premières victimes. Il y a cependant une association plus explicite qu'auparavant entre délinquance et prostitution de bas étage liée au fait que les deux phénomènes sont en augmentation au cours de cette période à Shanghai.

Si l'image des courtisanes avides d'argent tend à dominer les appréciations portées sur elles par les lecteurs, le Shen Bao rapporte aussi quelques anecdotes présentées comme véridiques qui nuancent ce portrait. Chen Xingtang, un commerçant ruiné par ses frasques dans les maisons de courtisanes est sorti de sa situation difficile grâce à une somme d'argent prêtée par une fille, elle-même sortie de la prostitution grâce à l'argent offert dans le passé par Chen. Depuis, elle s'est mariée et a fait des économies. C'est grâce à cette aide et à la promesse faite à cette courtisane de ne plus fréquenter les maisons de prostitution que le commerçant s'est de nouveau enrichi. Le message que véhicule cette histoire est ambivalent car les maisons de prostitution apparaissent comme des lieux où peuvent s'engloutir des fortunes, mais il projette aussi un portrait favorable de la courtisane qui a aidé le marchand ${ }^{31}$. Dans la même veine, un article raconte comment un lettré sans fortune, membre du cabinet d'un fonctionnaire, s'est résolu, sur les conseils de la courtisane qu'il fréquentait, à renoncer à cette relation et à partir. La fille, à qui il plaisait, ne lui faisait payer que les invitations extérieures (tangchai), mais lorsque le fonctionnaire auquel il était attaché a changé d'affectation, il a dû choisir entre rester auprès de la courtisane, sans ressources et au risque de devenir la risée des autres, et partir. La "morale" de l'histoire dégage un portrait positif de prostituée qui place ses sentiments avant l'argent ${ }^{32}$. Dans les deux cas, il s'agit de courtisanes.

19 Je n'ai trouvé qu'un seul article qui décrive avec sympathie mais de façon réaliste le sort des courtisanes et des prostituées. L'auteur rappelle qu'il ne faut pas se fier aux 
apparences, en particulier dans le cas des courtisanes dont les tenues et le confort sont trompeurs. Elles subissent comme les autres les humeurs des maquerelles, les caprices des clients et l'usure résultant d'horaires pénibles. Pour les prostituées ordinaires, il faut ajouter le temps passé à racoler dans les rues et les mauvais traitements. " De toutes les femmes, les prostituées sont celles qui ont le moins de valeur; de toutes les femmes qui souffrent, les prostituées sont celles qui souffrent le plus ». Cette citation rend bien compte de l'esprit de l'article qui appelle à plus de compassion à leur égard ${ }^{33}$.

L'effacement d'un système d'images

La tonalité dominante du discours des élites chinoises sur la prostitution au XIXe siècle apparaît étonnamment positive lorsqu'on se penche sur les ouvrages qu'elles rédigeaient et destinaient au fond à leur propre consommation. Elle ne représente pas un apport novateur dans ce qui constitue un mode d'expression traditionnel valorisant fortement les courtisanes. Dans la réalité, on l'a vu, les lettrés tenaient les prostituées ordinaires dans un profond mépris mêlé de dégoût. Néanmoins, comme cette facette a été presque complètement occultée au profit d'une imagerie qui parait les courtisanes de tous les talents, il n'a existé au sein de la culture chinoise qu'une seule perspective positive sur la prostitution qui consistait en une assimilation de l'ensemble du phénomène à sa strate supérieure. La société s'est donc imprégnée de cette conception "valorisante" de la prostitution, même si la réalité était bien différente. A travers ce phénomène d'assimilation entre courtisanes et prostitution, le discours des élites a déteint sur l'ensemble du corps prostitutionnel et contribué à faire de la prostitution une institution sociale "légitime". Naturellement, il faut aussi tenir compte des conceptions chinoises traditionnelles de la sexualité qui ne sont pas marquées par les inhibitions ou les tabous profonds qu'a véhiculés la culture judéo-chrétienne. Elles ont créé moins d'obstacle à l'insertion sociale de la prostitution qu'en Occident.

21 Le second point qui émerge dans le discours des élites, est leur inquiétude à l'égard de ce qu'elles perçoivent comme une montée du désordre social. La mutation rapide de la société et de l'économie locales provoque des phénomènes qui bousculent leurs habitudes et leurs conceptions "morales". Non pas que la prostitution populaire ait été dans le passé confinée strictement dans des lieux clos. Wang Tao évoque, dès le début des années 1850, la présence de prostituées "mobiles" (liuji) qu'il appelle les "dix mille fleurs" (wanhua) qui racolent dans les ruelles de la ville fortifiée et emmènent leurs clients dans de petites auberges ${ }^{34}$. Mais vingt ans plus tard, les élites sont confrontées à une extériorisation de plus en plus grande de la prostitution dans la ville, essentiellement dans les concessions étrangères, même si celle-ci ne prendra un caractère massif qu'au siècle suivant. La délinquance croissante les expose à de nouveaux risques. Ils voudraient endiguer le mouvement et regrettent l'envahissement d'un espace public où n'étaient tolérées auparavant que les courtisanes. Promenées d'un de ces lieux privilégiés de distraction des élites à un autre, en chaise à porteur, elles n'étaient pas cause de scandale public. Ce ne sont donc pas des considérations religieuses (sexualité) ou morales (sexualité vénale), même si cette dernière expression (daode) est parfois utilisée, qui motivent les lettrés, c'est ce qui "porte atteinte aux coutumes" (shang fenghua), c'est-à-dire à l'ordre public.

22 L'image des prostituées, y compris des courtisanes, s'est dégradée de façon continue jusqu'au XXe siècle. C'est le résultat d'un changement de sensibilité des élites mais aussi d'une transformation de ces élites elles-mêmes, déstructurées et renouvelées par le cours puissant de la modernisation qui se déroule à Shanghai. La culture 
traditionnelle des élites lettrées a été érodée et dans ce processus, les courtisanes ont perdu leurs "patrons" et ont été dépouillées de l'aura qu'ils leur avaient conférée. Les écrivains $d u$ tournant $d u$ siècle, pour la plupart installés dans les concessions étrangères, ont contribué par leurs romans au déclin de l'image de ce groupe, même si leur discours est parfois ambigu et si tout un genre, les "boussoles du monde galant", cherche encore à préserver l'illusion du passé. C'est un combat d'arrière-garde ou plutôt un chant du cygne. Les forces conjuguées de la critique sociale iconoclaste née du mouvement du 4 mai [1919], des revues féminines, des sciences sociales naissantes et d'une plus grande prise de conscience des questions sociales ont transformé la perception des femmes, des prostituées et des relations hommes-femmes dans la Chine urbaine. Les courtisanes n'ont pas échappé à la commercialisation des loisirs qui s'est développée dans les concessions étrangères de Shanghai et qui pour elles s'est traduite pas une sexualisation croissante et un déclassement vers la prostitution ordinaire.

L'évolution de l'image des prostituées dans les écrits des lettrés et dans la littérature est profondément révélatrice du changement des sensibilités. Il y a un renversement progressif d'optique, même si la vision positive "originale" véhiculée par le discours dominant des élites résultait d'un sérieux biais. Le monde enchanteur des courtisanes fait place à une prostitution frustre, caractérisée par l'appât de l'argent, les roueries, l'exploitation des filles, les maladies, la misère et la violence... Cette réalité n'est pas nouvelle; elle a toujours existé. L'infléchissement du discours est lié aux transformations du milieu de la prostitution lui-même, marqué par une forte expansion, une véritable "invasion" de l'espace public et une banalisation du fait prostitutionnel dans la vie quotidienne. Avant que cette transformation ne se produise, les élites avaient occulté dans leurs écrits la nature réelle de la prostitution, qu'il s'agisse des établissements populaires confinés en quelques lieux, peu visibles, ou des maisons de courtisanes, louées pour leur luxe et leur convivialité. À mesure que la prostitution grossit et s'expose, une réalité "nouvelle" commence à s'imposer. Les élites lettrées voient vaciller l'ordre économique et social qu'elles avaient dominé et qui marche vers sa désintégration. De nouvelles couches sociales émergent, moins sensibles aux valeurs éthiques et à la sensibilité esthétique des lettrés traditionnels. Les "nouveaux intellectuels" de la fin du XIXe siècle, prélude à la naissance des intellectuels modernes, trouvent dans la prostitution un thème privilégié d'expression de leur propre désarroi. L'image des courtisanes et du monde de la prostitution est sérieusement écornée dans leurs écrits. C'est l'amorce d'une brèche que ne vont cesser d'élargir les écrivains du XXe siècle, plus conscients de la prolétarisation de ce milieu.

La transformation de l'image de la prostitution à Shanghai n'est pas uniquement liée au changement social. L'étude des formes de discours sur la prostitution produits par les lettrés établit clairement que le développement de nouveaux canaux d'expression a apporté aussi un nouveau langage, de nouvelles représentations de la prostitution et une reconfiguration des perceptions sociales. Elle a mis en lumière des conceptions ou des idées qui n'étaient pas nécessairement nouvelles, mais qui étaient enfouies sous le vernis de l'expression littéraire traditionnelle. Ces conceptions ont finalement reçu une expression formelle dans les médias modernes. La presse qui s'est développée à la suite de la présence occidentale à Shanghai, en particulier dans les concessions étrangères, a ouvert un nouvel espace à la critique sociale. Le langage utilisé dans les journaux a une pertinence particulière : alors que les courtisanes étaient nommées soit par leur nom ou la catégorie à laquelle elles appartenaient (shuyu, changsan...) dans les écrits traditionnels de lettrés, elles se trouvent assimilées de manière croissante à la 
prostitution dans la presse à travers l'usage du mot commun $j i$ (prostituée). En d'autres termes, la ligne entre courtisanes et prostituées ordinaires devient floue, même si, selon le contenu des histoires, on peut discerner de quelle catégorie de prostituée il s'agit. Néanmoins, le langage des lettrés dans la presse tire toutes les classes de "femmes vénales » dans une seule catégorie générique. Les nouveaux médias qu'ils ont trouvés dans une ville comme Shanghai, notamment la presse, mais aussi le large marché fourni par un lectorat éduqué à la recherche d'une nouvelle identité ou de moyens d'échapper à la dure réalité, a créé un contexte qui reste unique à Shanghai pendant longtemps. De ce point de vue, les concessions étrangères ont été le berceau et le champ de bataille d'une nouvelle culture urbaine.

\section{BIBLIOGRAPHIE}

BAO Xiaoqun, 1988, « Xia Tingzhi he ta de 'Qinglouji' » (Xia Tingzhi et ses “Notes du pavillon vert”), Wenshi zhishi (Connaissances littéraires et historiques), $4:$ 83-85.

CHI Zhicheng, 1893, « Hu you mengying lu» (Notations et visions d'un voyage à Shanghai), Shanghai, Guji chubanshe, [rep.1989 "Hu you mengying lu" (Notations et visions d'un voyage à Shanghai), Dang'an yu lishi, $1: 1-7$.

Haishang qinglou lejing tushuo, 1892, (Les joies des pavillons verts de Shanghai, en mots et en images), Shanghai, s. éd.

Haishang qinglou tuji, 1892 (Notes et images des pavillons verts de Shanghai), Shanghai, Yingzhou xinzui shuwu, 4 volumes.

Haishang qunfangpu, 1884 (Annuaire des fleurs de Shanghai), Shanghai, Shenbao guan.

Haishang yeyou beilan, 1891 (Omnium du libertinage à Shanghai), Shanghai, s. éd., 2 volumes. HENRIOT Christian, 1980, « Le nouveau journalisme politique chinois, 1895-1911 », Chine. Cahiers d'Études Chinoises, Paris, INALCO, pp. 5-80.

- 1992, La Prostitution à Shanghai aux XIXe-XXe siècles (1849-1958), thèse de doctorat d'État, École des Hautes Études en Sciences Sociales, 3 volumes.

- 1992, « Medicine, V.D., and Prostitution in Pre-Revolutionary China », Social History of Medicine, Oxford University, $1: 95-120$.

- 1997, Belles de Shanghai. Prostitution et sexualité en Chine aux XIXe-XXe siècles, Paris, Editions du CNRS.

HERSHATTER Gail, 1997, Dangerous pleasures : Prostitution and Modernity in Twentieth Century Shanghai, Berkeley, University of California Press.

- 1992, "Courtesans and streetwalkers : The changing discourse on Shanghai prostitution, 1890-1949", Journal of the history of sexuality, $3: 2: 245-269$. 
« Hu chang yanjiu shumu tiyao », 1936 (Précis bibliographique pour la recherche sur les prostituées de Shanghai), in Shanghai yanjiu ziliao (Matériaux de recherche sur Shanghai), Shanghai, Shanghai tongshe, 579-608.

Hu shang xinhua baimei tu, 1884 (Gravures de cent nouvelles beautés de Shanghai), Shanghai. HUANG Kewu, 1988, «Cong Shen Bao yiyao guanggao kan min chu shanghai de yiliao wenhua yu shehui shenghuo, 1912-1926» (The Medical Culture and Social Life in Shanghai : A Study Based on the Medicine Advertisements in Shen Pao, 1912-1926), Jindaishi yanjiusuo jikan (Bulletin of the Institute of Modern History, Academia Sinica), XVII, part II : 141-194.

JUDGE Joan, 1996, Print and Politics : "Shibao" and the Culture of Reform in Late Qing China, Stanford, Stanford University Press.

LEVY Howard S., 1962, « The Gay Quarters of Ch'ang-an », Orient/West, 7-9 : 93-105.

- 1962, The Illusory Flame, Tokyo, Kenkyusha.

- 1963, « Record of the Gay Quarters », Orient/West, 8-5, sept -oct : 121-128 ; 8-6, nov -déc : 115-122 ; 9-1, janv -fév $1964:$ 103-110.

- 1966, A Feast of Mist and Flowers : The Gay Quarters of Nanking at the End of the Ming, Yokohama.

MEIJER Marinus Jan, 1980, « Slavery at the End of the Ch'ing Dynasty », in Cohen J., Chen-Chang F, Edwards R, Essays on China's Legal Tradition, Princeton, Princeton University Press : 327-358.

ROTOURS Robert, 1968, Courtisanes à la fin des T'ang entre circa 789 et le 8 janvier 881 : Pei-litche (Anecdotes du quartier du Nord) par Souen K’i, Paris, Presses Universitaires de France.

Shanghai pinyan baihua tu, 1884 (Cent portraits de courtisanes de Shanghai), Shanghai, 5 volumes.

Shen Bao Journal de Shanghai, quotidien, 1872-1949.

WAGNER Rudolf G., 1995, « The Role of the Foreign Community in the Chinese Public Sphere », China Quarterly 142 : $433 \mathrm{ff}$.

- 1999, «The Shenbao in Crisis: The International Environment and The Conflict Between Guo Songtao and the Shenbao », Late Imperial China, 20(1) : 107-138.

WALEY Arthur, 1967, « The Green Bower Collection » [1957] in The Secret History of the Mongols and Other Pieces, New York, 89-107.

WANG Tao, 1878, Huaguo jutan (Causerie sur le [théâtre du] monde des fleurs), Shanghai, Hanwen yuanshusi (rééd. 1929), pag. div et Songbin suohua (Propos futiles du bord de la rivière) Shanghai, s. éd.

WATSON James L., 1976, « Chattel Slavery in Chinese Peasant Society : A Comparative Analysis », Ethnology, 15 : 361-375.

- 1980, "Transactions in People: The Chinese Market in Slaves, Servants and Heirs ", in Watson, James L. (ed.), Asian and African Systems of Slavery, Berkeley, University of California Press : 223-250.

Xiangyan congshu, 1909-1911 (Collection [du monde] des beautés), édit. par Zhang Tinghua, Shanghai, Guoxue fulunshe, 80 volumes.

XIXI shanren, 1929, Wumen huafang lu (Récit des lupanars de Suzhou) in Yanshi shi er zhong, (Histoire de la lascivité en douze récits) édité par Yu Chensheng, Shanghai, Hanwenyuan shusi, 8 volumes (reproduction de l'édition de 1878). 
Yanshi shi er zhong, 1929 (Histoire de la lascivité en douze récits) édité par Yu Chensheng, Shanghai, Hanwenyuan shusi, (reproduction de l'édition de 1878), 8 volumes.

YEH Catherine Vance, 1997, « The life-style of four Wenren in late Qing Shanghai », Harvard Journal of Asiatic Studies, 57, 2 : 419-470.

YU Baosheng [pseud. de Wang Tao], 1929, Haizou yeyou lu (Récits de libertinage au bord de mer), Shanghai, Hanwen yuanshusi, rééd.

1929 Haizou yeyou fulu (Addenda aux récits de libertinage au bord de mer), Shanghai, Hanwen yuanshusi, rééd.

1929 Haizou yeyou yulu (Suite des récits de libertinage au bord de mer), Shanghai, Hanwen yuanshusi, rééd.

YU Huai, 1966, Banqiao zaji (Notes diverses du pont de bois), dont une version a été éditée par Levy, Howard S. (éd.), Banqiao zaji (Notes diverses du pont de bois) [1697], Yokohama, s. éd.

ZHU Ruiyue, 1990, « Shen bao fanying xia de shanghai shehui bianqian, 1895-1927 » (Social changes in Shanghai as reflected by the Shen Bao), M.A. thesis, Guoli taiwan daxue.

\section{NOTES}

1.Sur l'émergence de la presse politique à Shanghai, Henriot 1980. Plus récemment, plusieurs journaux ont été étudiés : Huang 1988 ; Zhu 1990 ; Judge 1996 ; Wagner 1999 et 1995.

2.J'ai étudié cette question dans deux chapitres non publiés de ma thèse d'Etat, en particulier le chapitre 16 « Droit, morale et esthétique », Henriot 1992. La dimension « représentation » de la prostitution est aussi abordée dans les travaux d'Henriot 1992 et 1997, d'Hershatter, 1992 et 1997 et Yeh 1997.

3.Yu Baosheng 1929, 1929b et 1929c.

4.Yu 1966, est un texte de la dynastie des Ming (1368-1644) qui décrit les courtisanes de Nankin ; Xixi 1929 (reproduction de l'édition de 1878), date du début du XIXe siècle et porte sur les courtisanes de Suzhou.

5.On trouve ce genre sous toutes les dynasties. Certains textes ont été traduits intégralement ou partiellement. [Song, 960-1127] Bao 1988; [Tang, 618-907] Rotours 1968 ; [Yuan, 1271-1368] Waley 1967 ; [Ming-Qing] Levy 1963, 1962, 1966 et 1962b. On trouvera une reproduction de bon nombre de ces textes dans deux compilations, Yanshi shi er zhong, (Histoire de la lascivité en douze récits) édité par Yu 1929 (reproduction de l'édition de 1878) et Xiangyan congshu [1909-1911].

6.Les deux autres ouvrages de Wang Tao, Wang [1878] et Wang s.d. sont écrits dans le même esprit et avec la même construction. C'est une juxtaposition de "libres propos" qui évoquent le passé et rapportent toutes sortes d'anecdotes ayant trait aux courtisanes. Songbin suohua évoque des événements plus divers, bien que plusieurs parties soient consacrées à la prostitution, à Shanghai, mais aussi à Canton ou au Japon. Quel que soit le lieu où Wang Tao a séjourné, il est resté un visiteur assidu des maisons de courtisanes ou, le cas échéant, de geishas. Huaguo jutan est une collection de biographies de courtisanes.

7.Chi 1893 et Chi Zhicheng 1989.

8.Une liste de ces ouvrages a été dressée en 1936 par les auteurs d'une collection de documents sur l'histoire de Shanghai. À cette époque, ils ont pu consulter la plupart des 
titres recensés dont une moitié se trouvait entre les mains d'un intellectuel connu, Xu Weinan. Je n'ai pas eu la même fortune, bien que j'aie fait le tour des bibliothèques susceptibles d'avoir ces ouvrages. Dans la mesure où il s'agit d'une sorte de bibliographie commentée, incorporant souvent la table des matières des livres cités, il est toutefois possible de se faire une idée du contenu. "Hu chang yanjiu shumu tiyao" 1936.

9.Shanghai pinyan baihua tu 1884 ; Hu shang xinhua baimei tu 1884 ; Haishang qunfangpu 1884 ; Haishang yeyou beilan 1891 ; Haishang qinglou lejing tushuo 1892 ; Haishang qinglou tuji 1892.

10.Je n'ai retrouvé que les titres cités dans cette étude, Haishang qinglou tuji 1892 ; Haishang yeyou beilan 1891.

11.Haishang qinglou tuji 1892.

12.Sur la prostitution populaire dans les écrits de Wang Tao, je ne fais que rendre compte ici de la vision globale qui se dessine à la lecture de ces textes. L'expression "squelette poudreux" (fen kulou) renvoie aussi à l'image d'êtres ayant perdu toute substance humaine en même temps que l'on peut y voir un jeu de mots, le terme "poudre" étant une des métaphores pour désigner les prostituées comme dans xiangfen (parfums et poudres) ou fenmian (visage poudré).

13.Chi Zhicheng $1893: 6$.

14.À propos d'une série d'articles sur les affaires impliquant des prostituées présentées devant la Cour mixte, le journal indique que Shanghai est devenu un centre de prostitution (yanhua zhi sou). Il note que bien que ces affaires soient d'un "faible intérêt", les cours des deux concessions ne peuvent pas ne pas en faire mention publiquement, comme pour les autres dossiers qu'elles traitent. Cette courte notation révèle précisément la faible importance accordée au phénomène prostitutionnel en dépit de son développement dans la ville. Shen Bao 21/10/1879.

15.Shen Bao 30/4/1872. Le calendrier occidental n'étant pas utilisé par le Shen Bao à cette époque, les dates sont indiquées d'après le calendrier traditionnel, soit le 27e jour du $7 \mathrm{e}$ mois lunaire.

16.Shen Bao 5/5/1872.

17.Shen Bao 6/2/1879. Wang Tao mentionne aussi l'existence d'un lieu dénommé maipochu (Office de vente d'épouses). C'était une sorte de prison où l'on enfermait les femmes adultères et où celles-ci étaient contraintes de se prostituer durant le temps de leur incarcération. Cet office « était supposé vendre ces femmes à des hommes de bonne volonté à la recherche d'une partenaire ». Les deux caractères guanmei (Entremetteur public) auraient été inscrits sur le mur arrière du bâtiment. Yu Baosheng, Haizou yeyou lu, p. III-3.

18.Sur l'esclavage et son abolition, voir Meijer 1980 ; Watson 1976 et 1980.

19.Shen Bao 17/10/1872.

20.Shen Bao 11/3/1873.

21.L'auteur ne donne pas de précisions sur l'origine et les lieux du débat. Il indique simplement une date imprécise, le début de l'ère Daoguang, soit 1821.

22.Shen Bao 9/5/1872.

23.Shen Bao 5/11/1872.

24.Le piantiwen est une sorte de "prose parallèle" qui fait alterner des séquences de quatre caractères avec des phrases de six caractères.

25.Shen Bao 27/7/1872.

26.Shen Bao 4/9/1872. 
27.Shen Bao 10/8/1872.

28.Les maladies vénériennes sont évoquées parmi d'autres facteurs sans mise en avant particulière, même si ces notations révèlent la conscience qu'ont les lettrés des effets de ces maladies sur l'hérédité. Mais ce n'est pas tant la maladie elle-même que le risque qu'elle fait encourir à la reproduction de la lignée qui est en cause. De toute manière, cette connaissance ne s'est pas traduite dans un discours cohérent et continu mettant en garde contre les infections vénériennes. Je n'ai trouvé qu'une lettre de lecteur exprimant un avis favorable à l'instauration d'un système de contrôle et de traitement médical des prostituées. Selon l'auteur, même si les filles se sentent humiliées par ces dispositions, ce n'est qu'une petite humiliation au regard des bénéfices à en attendre. Il considère que les clients ne font qu'attraper des infections vénériennes auprès d'elles car sans elles ils n'attraperaient rien [sic]. Les prostituées sont ici ravalées au rang de bétail, qu'il faut traiter. Le parallèle est frappant avec la condition des prostituées en Occident (Shen Bao 2/5/1873).

29.Shen Bao 27/5/1889.

30.Shen Bao 19/8/1899.

31.Shen Bao 4/4/1873.

32.Shen Bao 5/4/1873.

33.Shen Bao 28/3/1899.

34.Yu 1929 : II 4.

\section{RÉSUMÉS}

Cet article examine les formes du discours des lettrés chinois sur la prostitution de la fin du XIXe siècle à la Première Guerre mondiale. Il met en lumière le caractère ambivalent de ce discours qui, centré sur les courtisanes - la strate supérieure du monde de la prostitution - constitue une forme d'apologie ou de vision positive de la prostitution. Cette vision a pour partie imprégné la représentation collective de la prostitution en Chine. Toutefois cette représentation univoque masque un discours « en creux » clairement critique, voire méprisant, à l'égard de la prostitution ordinaire. Ce discours ambivalent a donné prise à une remise en cause progressive à mesure que l'évolution sociale et intellectuelle minait le statut des courtisanes et qu'une nouvelle vision issue des nouveaux média (essentiellement la presse) faisait de la prostitution un enjeu du débat public.

This paper examines the forms of discourse on prostitution by the Chinese literati from the end of the 19th century to the First World War. It highlights the ambivalent nature of a discourse that focused on the courtesans - the upper stratum of the world of prostitution - and, as a result, provided a kind of apology or positive view of prostitution. This vision has by and large permeated the collective perception of prostitution in Chinese society. This univocal discourse, however, overwrites another "sub-discourse" - one that appears by default - that is clearly critical, or even demeaning, on common prostitution. This ambivalent discourse was exposed to increasing criticisms as social and intellectual change undermined the status of courtesans and a new vision nurtured by the new media, especially the press, made prostitution an object of public debate. 


\section{AUTEUR}

\section{CHRISTIAN HENRIOT}

Christian HENRIOT est historien, spécialiste de la Chine contemporaine, à l'Université LumièreLyon 2 et directeur de l'Institut d'Asie Orientale (http://iao.ish-lyon.cnrs.fr). Il est l'auteur de plusieurs ouvrages (http://ghhat.univ-lyon2.fr/XXX) sur l'histoire politique et sociale de Shanghai, en particulier Shanghai 1927-1937. Elites locales et modernisation en Chine nationaliste, Paris, Editions de l'Ecole des Hautes Etudes en Sciences Sociales, 1991 ; Belles de Shanghai. Prostitution et sexualité en Chine aux XIXe-XXe siècles, Paris, Editions du CNRS, 1997 et Le Shanghai des années 30 (avec A. Roux), Paris, Editions Autrement, 1998. 